\title{
MAGNETIC FLUX DISTRIBUTION IN LOCALLY MAGNETIZED TWO-LAYER FERROMAGNETIC OBJECTS
}

\author{
V. N. Kostin ${ }^{1}$, O. N. Vasilenko ${ }^{1}$, A. M. Porseva ${ }^{2}$, A. A. Kabakova ${ }^{2}$, A. I. Melchakova ${ }^{2}$ \\ ${ }^{l}$ M.N. Miheev Institute of Metal Physics of Ural Branch of Russian Academy of Sciences, 18 S. Kovalevskoy st., \\ Ekaterinburg, Russian Federation \\ ${ }^{2}$ Ural Federal University named after the first President of Russia B.N.Yeltsin, 19 Mira st., \\ Ekaterinburg, Russian Federation
}

*Corresponding author. E-mail: kostin@imp.uran.ru; address for correspondence: 18, ul. S. Kovalevskoy, 620990, Ekaterinburg, Russian Federation. Tel.:+7 3433783659

Numerical simulation of the spatial distribution of the magnetic field and the flux in twolayer objects is executed. The possibility of measuring the depth of a high-coercive layer on a magnetically soft core by the value of the magnetic field measured on the surface of the object in the interpolar space of a U-shaped electromagnet is theoretically and experimentally shown.

Keywords: hardened layer, magnetic flux, field, numerical simulation, electromagnet.

DOI: $10.17804 / 2410-9908.2015 .5 .008-015$

\section{References}

1. Dalsky A.M., ed. Tekhnologiya konstruktsionnykh materialov. Uchebnik dlya studentov mashinostroitelnykh spetsialnostei vuzov, 5-e izd., ispravlennoe. [Technology of Structural Materials. Textbook]. M., Mashinostroenie Publ., 2004, 502 p. (In Russian).

2. Skhirtladze A.G., Yarushin S.G. Tekhnologicheskie protsessy v mashinostroenii. Uchebnik, 2-e izdanie, pererab. i dop. [Technological Processes in Mechanical Engineering. Textbook]. Stary Oskol, TNT Publ., 2008, 524 p. (In Russian).

3. Shcherbinin V.E., Gorkunov E.S. Magnitnyi Kontrol Kachestva Metallov [Magnetic Inspection of the Quality of Metals]. Ekaterinburg, UrO RAN Publ., 1996, 266 p. (In Russian).

4. Baev A.R., Mayorov A.L., Tischenko M.A. Ultrasonic Method for the Analysis of Metallic Materials Surface Hardening. Litye $i$ metallurgiya, no. 4, 2010, pp. 267-271. (In Russian).

5. Mikheev M.N. On the choice of optimum geometric dimensions of attached electromagnets of a coercimeter designed for testing the quality of heat and chemical-heat treatments of steel and cast-iron products, Fizika Metallov i Metallovedenie, 1957, vol. 5, iss. 1, pp. 44-52. (In Russian).

6. Mikheev M.N., Morozova V.M., Neizvestnov B.M., Surin G.V. Coercimeter with Attached Electromagnets, Defektoskopiya, 1969, no. 2, pp. 131-133. (In Russian).

7. Mikheev M.N., Gorkunov E.S. Magnitnye metody strukturnogo analiza i nerazrushayushchego kontrolya [Magnetic Methods of Structural Analysis and Nondestructive Testing]. M., Nauka Publ., 1993, 250 p. (In Russian).

8. Bida G.V., Mikheev M.N., Kostin V.N. Determination of the dimensions of attached electromagnets intended for the nondestructive inspection of depth and hardness of surface-hardened layers. The Soviet journal of nondestructive testing, 1984, vol. 20, iss. 8, pp. 495-501.

9. Mikheev M.N., Bida G.V., Kostin V.N., Mikhailova A.A., Kayukova T.N. Inspection of the depth and hardness of hardened layers after HF heating on crankshaft journals for motor cars. The Soviet journal of nondestructive testing, 1985, vol. 21, iss. 8, pp. 531-535.

10. Kostin V.N., Stashkov A.N., Nichipuruk A.P., Sapozhnikova Yu.G. Quality control of carburized parts made from 15XM steel. Russian Journal of Nondestructive Testing, 2004, vol. 40, iss. 12, pp. 826-829. DOI: 10.1007/s11181-005-0113-X.

11. Parallel'nye vychisleniya v UrO RAN. Zapusk programm iz paketa ANSYS. URL: http://www.parallel.uran.ru/node/264. 
12. Kostin V.N., Lukinykh O.N., Smorodinskii Ya.G., Kostin K.V. Simulation of field and inductance spatial distribution in locally magnetized massive objects and optimization of U-shaped transducer design. Russian Journal of Nondestructive Testing, 2010, vol. 46, iss. 6, pp. 403-410. DOI: $10.1134 / \mathrm{S} 1061830910060021$.

13. Vasilenko O.N., Kostin V.N. The topography of the field and flux inside and above the surfaces of ferromagnetic plates during their contact and contactless magnetization. Russian Journal of Nondestructive Testing, 2013, vol. 49, iss. 9, pp. 510-518. DOI: 10.1134/S1061830913090106.

14. Kostin V.N., Osintsev A.A., Stashkov A.N., Nichipuruk A.P., Kostin K.V., Sazhina E.Yu. Portable instruments for multiparameter magnetic evaluation of material structures. Russian Journal of Nondestructive Testing, 2008, vol. 44, iss. 4, pp. 280-289. DOI: 10.1134/S1061830908040086.

15. Kostin K.V., Kostin V.N., Smorodinskii Ya.G., Tsar'kova T.P., Somova V.M., Sazhina E.Yu. Choice of the parameters and algorithm for the magnetic hardness testing of thermally treated carbon steels by the method of regression modeling. Russian Journal of Nondestructive Testing, 2011, vol. 47, iss. 2, pp. 89-95. DOI: 10.1134/S1061830911020094.

16. Kostin K.V., Tsar'kova T.P., Nichipuruk A.P., Smorodinskii Ya.G. Measurement of the hysteresis characteristics of pipe steels under elastic and plastic tensile strain. Russian Journal of Nondestructive Testing, 2011, vol. 47, iss. 9, pp. 593-602. DOI: 10.1134/S1061830911090051. 
Подана в журнал: 18.09 .2015 г.

УДК 620.179.14

DOI: $10.17804 / 2410-9908.2015 .5 .008-015$

\title{
РАСПРЕДЕЛЕНИЕ МАГНИТНОГО ПОТОКА В ЛОКАЛЬНО НАМАГНИЧИВАЕМЫХ ДВУСЛОЙНЫХ ФЕРРОМАГНИТНЫХ ОБЪЕКТАХ
}

\author{
В. Н. Костин ${ }^{1 *}$, О. Н. Василенко ${ }^{1}$, А. М. Порсева ${ }^{2}$, А. А. Кабакова ${ }^{2}$, А. И. Мельчакова ${ }^{2}$ \\ ${ }^{1}$ Федеральное государственное бюджетное учреждение науки Институт физики металлов имени \\ М.Н. Михеева Уральского отделения Российской академии наук, ул. С. Ковалевской, 18, Екатеринбург, \\ Российская Федерачия \\ ${ }^{2}$ ФГАОУ ВПО «УрФУ имени первого Президента России Б.Н. Ельична», ул. Мира, 19, Екатеринбург, \\ Российская Федеращия
}

*Ответственный автор. Электронная почта: kostin@imp.uran.ru; адрес для переписки: 620990, ул. С. Ковалевской, 18, Екатеринбург, Российская Федерация. Телефон: +7 (343) 378-36-59

Выполнено численное моделирование пространственного распределения магнитных поля и потока в двуслойных объектах. Теоретически и экспериментально показана возможность измерения глубины высококоэрцитивного слоя на магнитомягкой сердцевине по величине магнитного поля, измеряемого на поверхности объекта в межполюсном пространстве П-образного электромагнита.

Ключевые слова: упрочненный слой, магнитный поток, поле, численное моделирование, электромагнит.

\section{1. Введение}

В современном машиностроении широко применяются различные методы поверхностного упрочнения стальных изделий $[1,2]$. Качество поверхностного упрочнения определяется, как правило, твердостью и глубиной упрочненного слоя. В некоторых случаях дополнительно требуется определять твердость неупрочненной сердцевины изделия [3].

Вследствие сложности и трудоемкости, а также невозможности контроля всех изготавливаемых изделий прямым, разрушающим способом задача косвенного, неразрушающего определения твердости и глубины упрочненных слоев до настоящего времени не потеряла своей актуальности. Имеются работы по ультразвуковому определению глубины упрочненного слоя [4], однако наибольшее практическое применение получила коэрцитиметрическая методика контроля поверхностного упрочнения [3, 5-10].

В коэрцитиметрической методике используются два приставных преобразователя, обладающих различными размерами и, соответственно, различной глубиной (объемом) промагничивания [3, 5-8]. О величине коэрцитивной силы, усредненной по всему намагничиваемому объему, судят по размагничивающему току в обмотках приставного электромагнита. Измерения обоими датчиками должны проводиться в одном и том же месте контролируемого объекта. Сначала для определения твердости упрочненного слоя проводят измерение относительной величины коэрцитивной силы с помощью преобразователя с малой глубиной промагничивания, которая должна быть меньше минимально необходимой глубины упрочненного слоя. Затем проводят измерения с помощью преобразователя с большой глубиной промагничивания, по результатам которых, с учетом показаний первого преобразователя, судят о глубине упрочненного слоя. Указанная методика является весьма продолжительной и трудоемкой. Её корректное применение возможно лишь в том случае, когда глубина и магнитные свойства слоя одинаковы в объеме, промагничиваемом наибольшим электромагнитом, что далеко не всегда выполняется на практике. Кроме того, датчик с малой глубиной промагничивания (т.е. с малым сечением полюсов и маленьким межполюсным расстоянием) 
является очень чувствительным к качеству контролируемой поверхности и зазорам в цепи “электромагнит-изделие” [8].

Разработка новых, более совершенных методик магнитного контроля поверхностного упрочнения требует исследования пространственного распределения магнитного потока при локальном намагничивании двуслойного ферромагнитного объекта, что является задачей настоящей работы.

\section{2. Образцы и методики исследований}

Исследование проводилось путем численного моделирования с использованием программы ANSYS [11]. Методика выполнения расчетов детально описана в работах [12, 13].

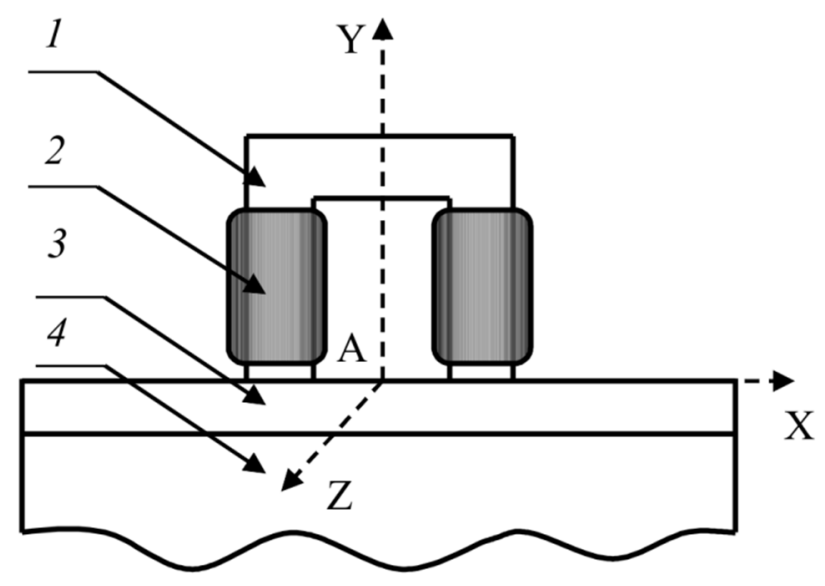

Рис. 1. Расчетная модель: 1 - магнитопровод; 2 - обмотки намагничивания; 3 - упрочненный слой; 4 - сердцевина изделия

На рис. 1 графически представлена модель локально намагничиваемого П-образным электромагнитом массивного двуслойного объекта. Магнитные свойства верхнего слоя, имитирующего поверхностное упрочнение, были следующими: $\mathrm{Hc}=35 \mathrm{~A} / \mathrm{cm}, \mu=40$. Толщина “упрочненного" слоя $D$ варьировалась в диапазоне от нуля до 15 мм.

Свойства нижнего слоя, имитирующего сердцевину изделия, были следующими:Нc= $5 \mathrm{~A} / \mathrm{cm}, \mu=200$. Размеры объекта контроля составляли: высота 230 мм, ширина 300 мм, толщина 56 мм. Размеры приставного электромагнита составляли: высота 100 мм, размер полюса $15 x 28$ мм. Магнитодвижущая сила равнялась 1800 Ампер-витков.

\section{3. Результаты и обсуждение}

На рис. 2 для различных значений глубины упрочненного слоя представлены распределения магнитных потока и поля в нейтральной плоскости электромагнита над поверхностью и внутри намагничиваемого объекта. Средняя плотность магнитного потока в “упрочненном" слое остается практически неизменной при увеличении $D$ от нуля до 15 мм (рис. $2 a$ ). Однако при $D \geq 5$ мм плотность магнитного потока становится неодинаковой на различных глубинах этого слоя, уменьшаясь от верхней к нижней границе. Это различие увеличивается с ростом толщины слоя $D$. При $D=10$ мм величина $B$ на поверхности и на нижней границе “упрочненного" слоя отличается примерно в два раза.

Из рис. 26 также можно увидеть, что при неизменной величине магнитодвижущей силы электромагнита поле на поверхности намагничиваемого объекта в середине межполюсного пространства (точка А) монотонно растет с ростом толщины “упрочненного" слоя. Соответствующая зависимость приведена на рис. 3 . 
$\mathrm{D}=0$
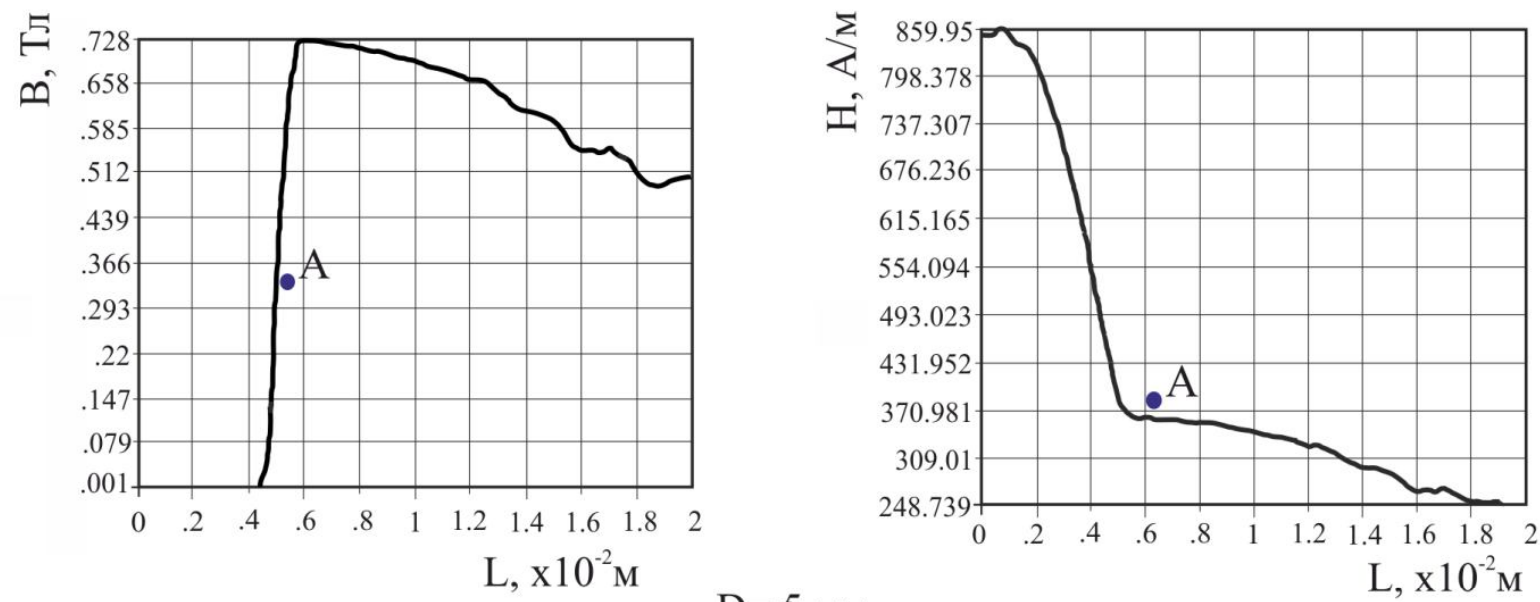

$\mathrm{D}=5 \mathrm{MM}$
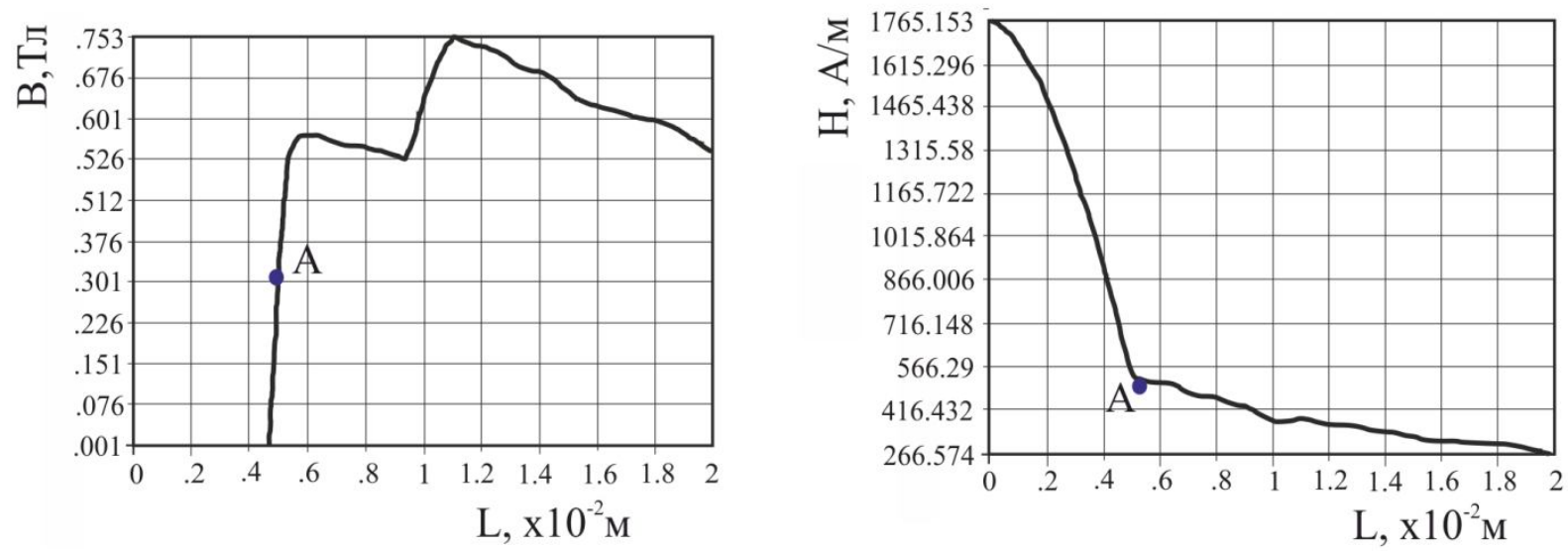

$\mathrm{D}=15 \mathrm{MM}$

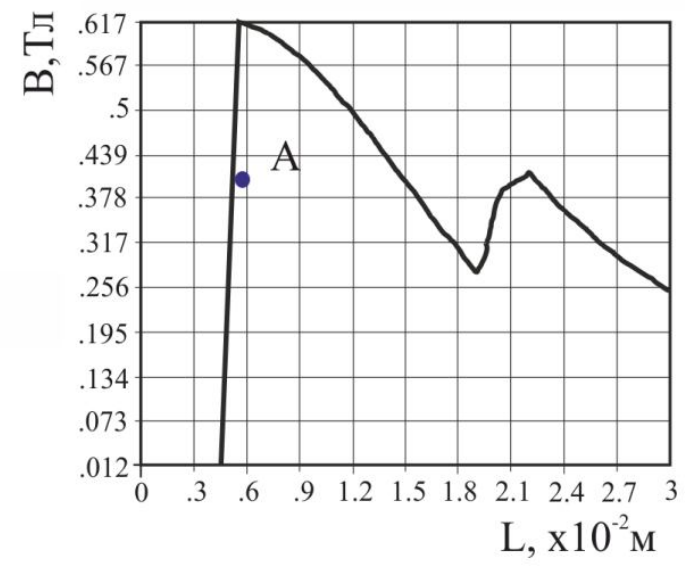

$a$

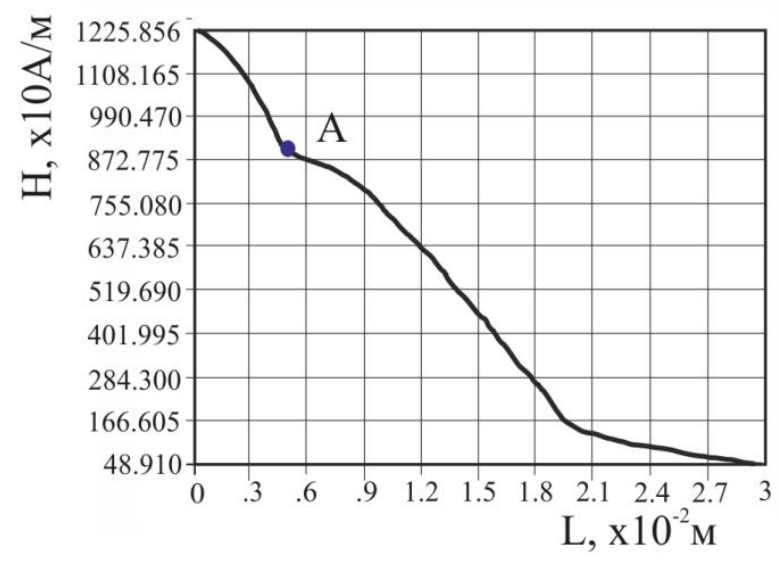

6

Рис. 2. Распределение плотности магнитного потока $(a)$ и напряженности магнитного поля (б) в нейтральной плоскости электромагнита над поверхностью (слева от точки А) и внутри (справа от точки А) намагничиваемых объектов с различной глубиной “упрочненного” слоя D

Согласно расчетам, поле $H_{A}$ практически линейно растет на поверхности объекта при возрастании величины $D$ от 2 до 15 мм, увеличиваясь более, чем в 2 раза. Таким образом, поле $H_{A}$ является потенциальным параметром контроля глубины упрочненного слоя, причем даже тогда, когда величина $D$ превышает толщину полюса электромагнита. Однако измерение параметров кривой намагничивания, к которым относится величина $H_{A}$, на практи- 
ке весьма затруднено в связи с невозможностью качественного размагничивания крупногабаритных изделий и изделий сложной формы.

Для определения более подходящего параметра контроля с помощью прибора СИМTECT $[14,15]$ было проведено измерение напряженности поля $H_{p}$, которое достигалось в середине межполюсного пространства (точка $A$ ) на поверхности двуслойных объектов после их намагничивания П-образным электромагнитом и последующего перемагничивания по нисходящей ветви петли гистерезиса до фиксированного значения магнитного потока [16]. Упрочненный слой составлялся из шлифованных пластин различной толщины, изготовленных из закалённой стали $62 \mathrm{C} 2$. Сердцевину представляла собой шлифованная плита с размерами 100x170x34 мм, изготовленная из отожженной стали 3. Полученные экспериментальные результаты представлены на рис. 4.

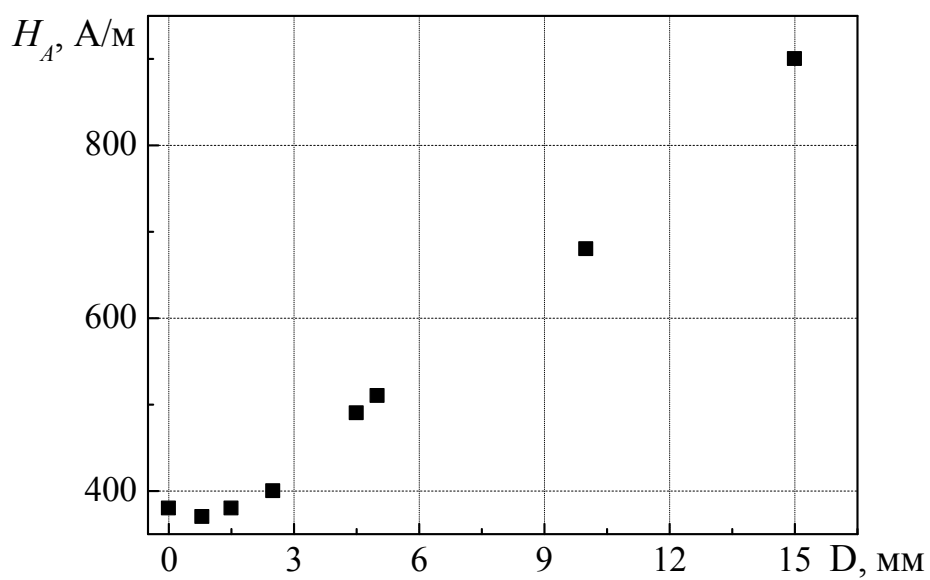

Рис. 3. Расчетная зависимость напряженности магнитного поля в межполюсном пространстве электромагнита на поверхности двуслойного объекта от толщины “упрочненного” слоя при фиксированной магнитодвижущей силе

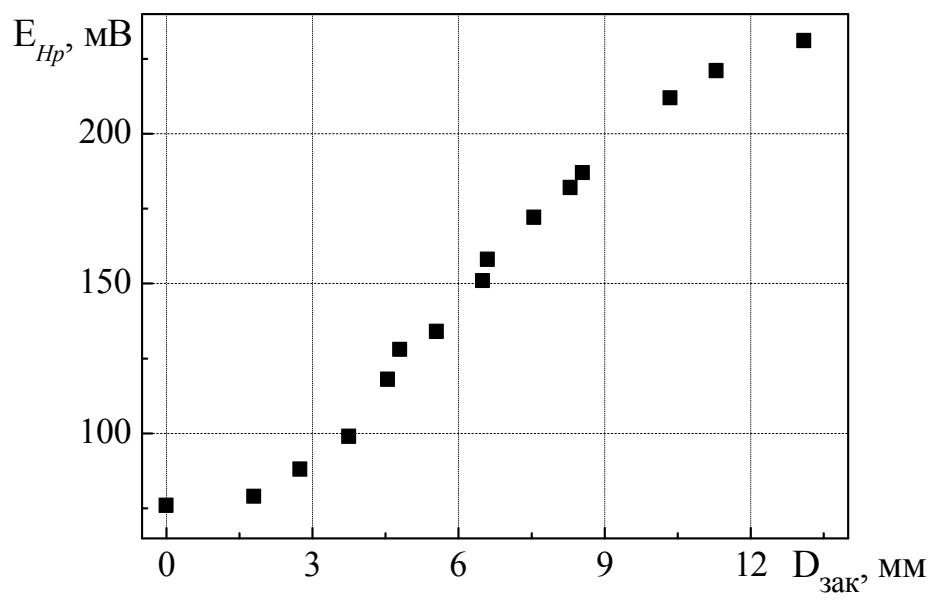

Рис. 4. Зависимость относительной величины напряженности магнитного поля на поверхности двуслойного объекта, после его перемагничивания по нисходящей ветви петли гистере-

зиса до фиксированного значения магнитного потока, от толщины закаленного слоя $\mathrm{D}_{\text {зак }}$

Как следует из полученных результатов, поле $H_{p}$ коррелирует с рассчитанным полем $H_{A}$, однако в отличие от последнего может быть легко измерено с помощью существующих измерительных устройств $[14,15]$. Таким образом, возможен контроль глубины упрочненно- 
го слоя по величине магнитного поля, измеряемого на поверхности локально намагничиваемого объекта контроля.

\section{4. Заключение}

Численным моделированием и экспериментально установлена возможность определения глубины упрочненного слоя на поверхности массивной магнитомягкой сердцевины по величине магнитного поля на поверхности локально намагничиваемого объекта. При этом, в отличие от известной коэрцитиметрической методики [3], диапазон контролируемых толщин может превышать толщину полюсов приставного электромагнита.

Весьма полезной была бы разработка методики и аппаратуры, в которых для селективного определения параметров упрочненного слоя использовалось бы экспрессное измерение необходимых магнитных параметров контроля с помощью единственного преобразователя. Решение этой задачи требует дальнейших исследований.

\section{Благодарность}

Работа выполнена по программе фундаментальных исследований УрО РАН 2015-2017 гг. проект 15-17-2-5.

\section{Литература}

1. Технология конструкционных материалов. Учебник для студентов машиностроительных специальностей вузов / А. М. Дальский, Т. М. Барсукова, Л. Н. Бухаркин и др. / Под ред. А. М. Дальского. - 5-е изд., испр. - М. : Машиностроение, 2004. - С. 295-441.

2. Схиртладзе А. Г., Ярушин С. Г. Технологические процессы в машиностроении: учебник. - 2-е изд., перераб. и доп. - Старый Оскол : ТНТ, 2008. - 524 с.

3. Щербинин В. Е., Горкунов Э. С. Магнитные методы структурного анализа и неразрушающего контроля. - Екатеринбург : Изд-во УрО РАН, 1996. - 266 с.

4. Баев А. Р., Майоров А. Л., Тищенко М. А. Ультразвуковой метод анализа поверхностного упрочнения металлических изделий // Литье и металлургия. - 2010. - № 4 (58). - C. 267-271.

5. Михеев М. Н. О выборе оптимальных геометрических размеров приставных электромагнитов коэрцитиметра, предназначенного для контроля качества термической и химикотермической обработки стальных и чугунных изделий // ФММ. - 1957. - Т. 5, вып. 1. - C. 44-52.

6. Михеев М. Н., Морозова В. М., Неизвестнов Б. М., Сурин Г. В. Коэрцитиметры с приставными электромагнитами // Дефектоскопия. - 1969. - № 2. - С. 131-133.

7. Михеев М. Н., Горкунов Э. С. Магнитные методы структурного анализа и неразрушающего контроля. - М. : Наука, 1993. - 250 с.

8. Bida G. V., Mikheev M. N., Kostin V. N. Determination of the dimensions of attached electromagnets intended for the nondestructive inspection of depth and hardness of surface-hardened layers // SJNDT. - 1984. - Vol. 20, iss. 8. - P. 495-501.

9. Inspection of the depth and hardness of hardened layers after HF-heating on crankshaft journals for motor cars / M. N. Mikheev, G. V. Bida, V. N. Kostin, A. A. Mikhailova, T. N. Kayukova // SJNDT. - 1985. - Vol. 21, iss. 8. - P. 531-535.

10. Quality control of carburized parts made from 15XM steel / V. N. Kostin, A. N. Stashkov, A. P. Nichipuruk, Yu. G. Sapozhnikova // RJNDT. - 2004. - Vol. 40, iss. 12. - P. 826-829. DOI: $10.1007 / \mathrm{s} 11181-005-0113-\mathrm{x}$.

11. Параллельные вычисления в УрО РАН. Запуск программ из пакета ANSYS. URL: http://www.parallel.uran.ru/node/264.

12. Simulation of field and inductance spatial distribution in locally magnetized massive objects and optimization of U-shaped transducer design / V. N. Kostin, O. N. Lukinykh, 
open-aCcess journal

Ya. G. Smorodinskii // RJNDT. - 2010. - Vol. 46, iss. 6. - P. 403-410. DOI: $10.1134 / \mathrm{S} 1061830910060021$.

13. Vasilenko O. N., Kostin V. N. The topography of the field and flux inside and above the surfaces of ferromagnetic plates during their contact and contactless magnetization // RJNDT. - 2013. - Vol. 49, iss. 9. - P. 510-518. - DOI: 10.1134/S1061830913090106.

14. Portable instruments for multiparameter magnetic evaluation of material structures / V. N. Kostin, A. A. Osintsev, A. N. Stashkov, A. P. Nichipuruk, K. V. Kostin, E. Yu. Sazhina // RJNDT. - 2008. - Vol. 44, iss. 4. - P. 280-289. - DOI: 10.1134/S1061830908040086.

15. Choice of the parameters and algorithm for the magnetic hardness testing of thermally treated carbon steels by the method of regression modeling / K. V. Kostin, V. N. Kostin, Ya. G. Smorodinskii, T. P. Tsar'kova, V. M. Somova, E. Yu. Sazhina // RJNDT. - 2011. - Vol. 47, iss. 2. - P. 89-95. - DOI: 10.1134/S1061830911020094.

16. Measurement of the hysteresis characteristics of pipe steels under elastic and plastic tensile strain / K. V. Kostin,. T. P. Tsar'kova, A. P. Nichipuruk, Ya. G. Smorodinskii // RJNDT. - 2011. Vol. 47, iss. 9. - P. 593-602. - DOI: 10.1134/S10618309110. 